\title{
EFEITOS TARDIOS DO BAIXO PESO AO NASCER ${ }^{+}$
}

\section{LATE EFFECTS OF LOW BIRTH WEIGHT}

Elizabeth Maria Bismarck-Nasr**
Maria Fernanda Petroli Frutuoso**
Ana Maria Dianezi Gamabardella**

Bismarck-Nasr EM, Frutuoso MFP, Gamabardella AMD. Efeitos tardios do baixo peso ao nascer. Rev Bras Crescimento Desenvol Hum. 2008; 18(1): 98-103.

Resumo: Estudos recentes sugerem que doenças como diabetes tipo 2, coronariopatias, hipertensão arterial e obesidade, relacionam-se a um inadequado crescimento intra- uterino, fenômeno denominado programação ou hipótese da origem fetal de doenças. Deste modo, a nutrição durante o período gestacional mostra-se como um importante campo para investigação científica, uma vez que o baixo peso ao nascer ainda apresenta-se como importante problema de saúde pública em países em desenvolvimento e que, grande porção destas nações vivencia o processo denominado transição nutricional, onde mudanças econômicas, nutricionais e de estilo de vida permitem maior desenvolvimento de obesidade.

Palavras-chave: Baixo peso ao nascer; obesidade; programação.

Estudos recentes sugerem que doenças como diabetes tipo 2, coronariopatias, hipertensão arterial e obesidade, relacionam-se a um inadequado crescimento intra- uterino, fenômeno denominado programação ou hipótese da origem fetal de doenças ${ }^{1-5}$.

Entende-se por programação a modificação permanente na estrutura, fisiologia ou metabolismo de um órgão devido a estímulos ou agravos durante um período crítico de desenvolvimento $^{6,7}$. Um dos mais importantes tipos de programação consiste naquele induzido pela nutrição no início da vida, sendo o baixo peso ao nascer um marcador de deficiente nutrição fetal ${ }^{1}$.

No caso da desnutrição intra -uterina, o feto desenvolve mecanismos de adaptação metabólicos e endócrinos para sua sobrevivência em situações de restrição nutricional, no entanto, após o nascimento, com a normalização da oferta de alimentos, esta adaptação pode acarretar efeitos deletérios em longo prazo, como obesidade e intolerância à glicose $\mathrm{e}^{2,8}$.

A hipótese da origem fetal de doenças parece relacionar-se ao crescimento intra-uterino restrito e não apenas ao baixo peso ao nascer, desse modo, bebês prematuros com peso adequado para idade gestacional estariam protegidos de complicações futuras ${ }^{2}$.

Os primeiros achados epidemiológicos sobre a hipótese de que restrições nutricionais

\footnotetext{
* $\quad$ Mestre em Nutrição em Saúde Pública pela Faculdade de Saúde Pública/ USP. Nutricionista responsável técnica pelo Programa Municipal de Nutrição da Secretaria de Saúde do Município de São José dos Campos - SP.

Av Cidade Jardim, 3141, casa 47. Quinta das Flores, São José dos Campos, SP. CEP 12233-002 fone: 12- 39164203/ 12- 81156332 - e-mail: beth_bismarck@yahoo.com

** Doutora em Nutrição em Saúde Pública pela Faculdade de Saúde Pública/ USP. Professora Doutora do curso de graduação em Nutrição da Universidade Anhembi-Morumbi e Universidade de São Francisco de Bragança.

*** Professora Doutora do Departamento de Nutrição da Faculdade de Saúde Pública da USP. Av. Dr. Arnaldo, 715 - Cerqueira César- São Paulo, SP. CEP 01246-904.

+ Artigo baseado na Dissertação de Mestrado apresentada ao Departamento de Nutrição da Faculdade de Saúde Pública da FSP.
} 
durante o período gestacional acarretariam alterações metabólicas irreversíveis no feto surgiram a partir de estudos sobre a "fome holandesa" durante o final da Segunda Guerra Mundial, quando o governo alemão passou a diminuir a oferta calórica destinada aos residentes no nordeste da Holanda. No período entre $\mathrm{Ou}-$ tubro de 1944 e Janeiro de 1945 o consumo energético destes indivíduos caiu de aproximadamente $1500 \mathrm{kcal}$ para $1000 \mathrm{kcal}$ e de Abril a Maio de 1945; esta cota representava cerca de $500 \mathrm{kcal}$, quando ocorreu a libertação da Holanda, finalizando este período9.

A partir deste fato, diversas coortes passaram a ser estudadas, conforme período de crescimento intra-uterino e restrição calórica. Maior prevalência de obesidade, aos 18 anos foi observada nos rapazes que sofreram restrições alimentares nos dois primeiros trimestres de gestação, período em que o hipotálamo começa a se organizar. Por outro lado, os jovens expostos à "fome" no último trimestre de gestação, período caracterizado pela multiplicação dos adipócitos e rápido incremento de gordura corporal, não apresentaram obesidade aos 18 anos, portanto, o período gestacional em que ocorre o agravo nutricional parece determinar a predisposição à obesidade futura? .

Outra análise, também realizada com este grupo de indivíduos nascidos durante a "fome holandesa", detectou nas mulheres valores de índice de massa corporal (IMC) e circunferência da cintura significativamente maiores, aos 50 anos de idade, quando comparados aos dados antropométricos de mulheres que não sofreram restrições nutricionais no período intra -uterino ${ }^{10}$.

Resultados de estudo prospectivo finlandês verificaram maior incidência de morbi -mortalidade devido a doenças cardiovasculares na idade adulta entre mulheres que apresentaram comprometimento linear ${ }^{11}$ e homens magros com baixo índice ponderal ${ }^{12}$. Os autores apontaram para diferenças entre os sexos quanto ao tipo de desnutrição ao nascer, sendo que o risco cardiovascular nas mulheres aumentou em 10,2\% para cada centímetro a menos no comprimento ao nascer $^{11}$ e para os homens, aumentou em $14 \%$ para cada unidade a menos no índice ponderal ${ }^{12}$.
Outro achado interessante, os homens que morreram por doenças cardíacas apresentaram valores de índice de massa corporal acima da média do grupo estudado durante o período dos 7 aos 15 anos de idade, sendo que, aos 11 anos, os meninos apresentaram risco aumentado em $22 \%$ para cada unidade a mais de IMC. Vale ressaltar que os meninos com alto índice ponderal ao nascer mostraram risco diminuído para doenças cardiovasculares, mesmo quando obesos durante a adolescência ${ }^{12}$.

Explorando um pouco mais os resultados deste seguimento finlandês, este grupo de pesquisadores detectou que a intensidade da velocidade de recuperação nutricional durante a infância e início da puberdade, principalmente referente ao ganho de peso, consistiu um importante precursor de diabetes tipo 2, hipertensão arterial e doenças cardiovasculares no futuro destes indivíduos, sendo que o risco para estas doenças mostrou-se elevado conforme aumento dos valores de IMC aos 11 anos de idade ${ }^{13}$.

Estudo comparativo entre crianças com diagnóstico de baixa estatura e eutróficas, com idade entre 8 e 11 anos, residentes em favelas no Município de São Paulo, verificou alterações metabólicas quanto à redução na oxidação lipídica no período pós-prandial, nas crianças com baixa estatura, aspecto que poderia favorecer acúmulo de gordura corporal neste grupo, mostrando consistência à teoria de que a desnutrição no início da vida acarretaria modificações metabólicas permanentes ${ }^{14}$.

Após 36 meses, este mesmo grupo de indivíduos recebeu reavaliação do estado nutricional e os resultados obtidos confirmaram os achados do estudo anterior. Os adolescentes do sexo masculino (idade entre 11 e 15 anos) com baixa estatura apresentaram incremento maior na porcentagem de gordura corporal e menor na quantidade relativa de massa magra quando comparados aos adolescentes eutróficos, tais achados foram medidos por meio de dupla emissão de feixes de raios-X (DEXA) ${ }^{15}$.

Os achados descritos acima mostram-se consistentes com a proposta sugerida por Frisancho $^{16}$. Segundo este autor, indivíduos expostos à desnutrição intra-útero e durante os pri- 
meiros anos de vida, apresentam redução no metabolismo oxidativo de lipídios, utilizando carboidratos como fonte preferencial para obtenção de energia, resultando em maior depósito de gordura corporal, contribuindo para o aumento na prevalência de obesidade nas nações em desenvolvimento.

Por outro lado, alguns estudiosos referem que os efeitos tardios do baixo peso ao nascer derivam de um reduzido conteúdo de massa muscular, acarretando baixa atividade metabólica ${ }^{17,18,19}$ que, associada ao tipo de alimentação hipercalórica, típica da adolescência, favoreceria o acúmulo de gordura corporal, e isto explicaria o fato destes indivíduos apresentarem obesidade na idade adulta ${ }^{19}$.

Algumas hipóteses plausíveis que justificariam a associação entre desnutrição intra -uterina e redução na proporção de massa magra no futuro foram levantadas, tais como, favorecimento para o desenvolvimento de órgãos vitais, como o cérebro, em detrimento ao crescimento muscular em situações de estresse nutricional durante o período gestacional; hipoglicemia fetal decorrente de fornecimento insuficiente de glicose, acarretando reduzida secreção de insulina, que conseqüentemente, estimularia o catabolismo protéico; a má nutrição fetal reduziria a concentração do fator de crescimento insulino -dependente (IGF-1), comprometendo o crescimento da massa muscular ${ }^{19}$.

Como o músculo consiste num importante local para captação de glicose devido à ação da insulina, a programação de uma reduzida proporção de massa magra provocaria resistência à insulina, representando um dos possíveis mecanismos que relacionam o baixo peso ao nascer com risco elevado para doenças cardiovasculares no futuro ${ }^{17,19}$.

De fato, resultados de uma coorte de crianças norte -americanas (NHANES III, 19881994), acompanhadas dos 2 aos 47 meses de idade, mostraram menor conteúdo de massa muscular entre aquelas que nasceram com baixo peso, independentemente da idade gestacional ${ }^{20}$.

Deve-se considerar a possibilidade de que a desnutrição intra -uterina acarretaria em efeitos adversos à saúde em longo prazo, uma vez que o baixo peso ao nascer ainda apresenta-se como importante problema de saúde pública em países em desenvolvimento, e que grande porção destas nações experimentam o processo denominado transição nutricional, onde mudanças econômicas, nutricionais e de estilo de vida, sobretudo referentes ao sedentarismo, permitem rápido crescimento pós-natal, predispondo estes indivíduos ao desenvolvimento da síndrome plurimetabólica ${ }^{7,8,17,21}$.

Vários são os achados que relacionam o ambiente gestacional com efeitos tardios na composição corporal dos indivíduos, evidenciando a fase gestacional como um período crítico para a gênese da obesidade. $\mathrm{O}$ modo pelo qual a memória metabólica incorporada durante a exposição intra-útero prejudica a saúde destes indivíduos representa um desafio fascinante para o meio científico ${ }^{22}$.

No entanto, estudos referentes à hipótese da programação geralmente consideram apenas dois momentos para verificar a relação entre $o$ estado nutricional ao nascer e atual. Na ausência de uma terceira informação antropométrica, intermediária, torna-se difícil distinguir a interferência do peso ao nascer na prevalência de excesso de peso corporal atual.

Outro aspecto importante a ser considerado seria a avaliação do estado nutricional materno durante a gestação dos indivíduos estudados. Resultados de um estudo longitudinal conduzido nas Filipinas sugerem que a desnutrição materna no final do período gestacional e sua conseqüente oferta inadequada de nutrientes ao feto parece interferir com maior intensidade na predisposição ao desenvolvimento de doenças cardiovasculares durante a adolescência quando comparada ao peso de nascimento ${ }^{23}$. Estes autores identificaram perfil lipídico com maior potencial aterogênico entre os adolescentes do sexo masculino que sofreram restrições nutricionais no final da gestação.

Além disso, a elevada velocidade de crescimento pós-natal e o tipo de alimentação oferecido durante o período neonatal também parecem interferir na predisposição ao desenvolvimento de doenças crônicas no futuro ${ }^{24,25,26}$.

Estudos longitudinais realizados nas Ilhas 
Seychelles, país localizado no Oceano Índico e em intenso processo de transição nutricional ${ }^{24} \mathrm{e}$ na Islândia ${ }^{25}$ verificaram que o elevado ganho de peso no primeiro ano de vida associou-se significativamente à prevalência de sobrepeso e obesidade na infância e adolescência.

Um grupo de pesquisadores que compararam bebês prematuros que receberam fórmulas infantis para pré-termos com aqueles alimentados com leite humano ou fórmulas infantis tradicionais, verificou resultados desfavoráveis em relação ao perfil lipídico sanguíneo, pressão arterial, resistência à leptina e à insulina durante $\mathrm{a}$ infância e adolescência nos bebês que receberam fórmula infantil com maior densidade energética e protéica (fórmula para prematuros) e que, conseqüentemente, apresentaram maior crescimento pós-natal ${ }^{27-29}$.

Segundo estes autores, devido ao fato de bebês com crescimento intra-uterino restrito mostrarem rápido crescimento pós-natal, as associações entre baixo peso ao nascer e doenças crônicas no futuro poderiam sinalizar, na realidade, as consequiências decorrentes dos efeitos adversos de uma aceleração no crescimento e não de restrições nutricionais no período gestacional ${ }^{26}$.

Segundo Gillman ${ }^{30}$, parecem existir três possíveis inferências a partir de estudos que analisaram o aumento no risco para o desenvolvimento de doenças cardiovasculares tanto em indivíduos que nasceram pequenos para idade gestacional quanto naqueles que adquiriram excesso de peso corporal no decorrer da vida. A pri- meira refere-se ao aumento no risco para o desenvolvimento de doenças crônicas no futuro devido a um rápido crescimento pós-natal. A segunda inferência aponta para os riscos oriundos de um crescimento intra-uterino restrito e a terceira representa uma interação entre as duas primeiras inferências. Por este motivo, o autor sugere que estudos sobre a origem fetal de doenças contemplem diversas avaliações antropométricas ao longo da infância, adolescência e vida adulta, permitindo a diferenciação entre influências pré-natais e decorrentes de um rápido crescimento em períodos críticos para o desenvolvimento da obesidade.

Estudos epidemiológicos e experimentais oferecem fortes evidências de que os efeitos da programação interferem na gênese de doenças na idade adulta, sendo este fato particularmente importante em nações que experimentam o processo de transição nutricional. Por este motivo, as políticas de saúde devem intensificar seus esforços em medidas que promovam adequado ganho de peso intra-uterino e nutrição pós-natal, estimulando a prática de aleitamento materno. Evitando, deste modo, dilemas como promover ou não a rápida recuperação do estado nutricional durante a infância, principalmente com intervenções nutricionais que proporcionem maior ganho de peso corporal do que de estatura, refletindo em aumento de gordura corporal, como antigos programas de alimentação e nutrição que preconizavam a distribuição de alimentos para enriquecimento do valor calórico da dieta oferecida ao público infantil ${ }^{31}$.

\begin{abstract}
Recent studies suggest that diseases such as Type 2 diabetes, coronary diseases, hypertension and obesity are related to inadequate gestational development. Such phenomenon is known as programming or hypothesis of the fetal origin of disease. Therefore, the nutrition status during gestational period is an important field of scientific research, specially considering that low birth weight is an important public health problem in developing countries, which are subject to the process known as nutritional transition, where economic, nutritional and lifestyle changes allow a higher rate of obesity development.
\end{abstract}

Keywords: Low birth weight; obesity; programming. 


\section{REFERÊNCIAS}

1. Lucas, A; Fewtrell, MS; Cole, TJ. Fetal origins of adult disease - the hypotesis revisited. BMJ 1999; 319: 245-249.

2. Godfrey, KM \& Barker, DJP. Fetal nutrition and adult disease. Am J Clin Nutr 2000; 71 (suppl): 1344S-1352S.

3. Iliadou, A; Cnattingius, S; Lichtenstein, P. Low birth weight and type 2 diabetes: a study on 11162 swedish twins. Int J Epidemiol 2004; 33: 948-953.

4. Sawaya, A.L. Desnutrição: conseqüências em longo prazo e efeitos da recuperação nutricional. Estudos Avançados 2006; 20 (58): 147-158.

5. Gluckman, PD; Seng, CY; Fukuoka, H; Beedle, AS; Hanson, MA. Low birthweight and subsequent obesity in Japan. Lancet 2007; 369: 1081-1082.

6. Lucas, A. Role of nutritional programming in determining adult morbidity. Arch Dis Child 1994; 71:288-290.

7. Law, C. Adult obesity and growth in childhood. BMJ 2001; 323: 1320-1321.

8. Robinson, R. The fetal origins of adult disease. BMJ 2001; 322: 375-376.

9. Ravelli, GP; Stein, Z; Susser, M. obesity in young men after famine exposure in utero and early infancy. N Engl J Med 1976; 259: 349-353.

10. Ravelli, ACJ; Meulen, JHPD; Osmond, C; Barker, DJP; Bleker, OP. Obesity at the age of $50 \mathrm{y}$ in men and women exposed to famine prenatally. Am J Clin Nutr 1999; 70: 811-816.

11. Forsén, T; Eriksson, JG; Tuomilehto, J; Osmond, C; Barker, DJP. Growth in utero and during childhood among women who develop coronary heart disease: longitudinal study. BMJ 1999; 319 : 1403-1407.

12. Eriksson, JG; Forsén, T; Tuomilehto, J; Winter, PD; Osmond, C; Barker, DJP. Catch- up growth in childhood and death from coronary heart disease: longitudinal study. BMJ 1999; 318: 427-431.

13. Barker, DJP; Eriksson, JG; Forsén, T; Osmond, C. Fetal origins of adult disease: strength of effects and biological basis. Int J Epidemiol 2002; 31: 1235-1239.

14. Hoffman, DJ; Sawaya, AL; Verreschi, I; Tucker, KL; Roberts, SB. Why are nutritionally stunted children at increased risk of obesity? Studies of metabolic rate and fat oxidation in shantytown children from São Paulo, Brazil. Am J Clin Nutr 2000; 72: 702-707.

15. Martins, P. A.; Hoffman, D. ; Fernandes, M. T. ; Nascimento, C. ; Roberts, S. B. ; Sesso, R. ;
Sawaya, A. L. Stunted children gain less lean body mass and more fat mass than their non-stunted counterparts: a prospective study. Br J Nutr 2004; 92: 819-825.

16. Frisancho, AR. Reduced rate of fat oxidation: a metabolic pathway to obesity in the developing nations. Am J Hum Biol 2003; 15: 522-532.

17. Adair, LS. Early nutrition conditions and later risk of disease. In: Caballero, B \& Popkin, BM. The nutrition transition: diet and disease in the developing world. London, Academic Press, 2002.

18. Ben-Shlomo, Y \& Kuh, D. A life course approach to chronic disease epidemiology: conceptual models, empirical challenges and interdisciplinary perspectives. Int J Epidemiol 2002; 31: 285-293.

19. Singhal, A; Wells, J; Cole, TJ; Fewtrell, M; Lucas, A. Programming of lean body mass: a link between birth weight, obesity, and cardiovascular disease. Am J Clin Nutr 2003; 77: 726-730.

20. Hediger, ML; Overpeck, MD; Kuczmarski, RJ; McGlynn, A; Maurer, KR; Davis, W. Muscularity and fatness of infants and young children born small- or large- for- gestational- age. Pediatrics 1998; 102 (5). URL: http://www.pediatrics.org/cgi/ content/full/102/5/e60

21. Popkin, BM. An overview on the nutrition transition and its health implications: the Bellagio meeting. Public Health Nutr 2002; 5(1A): 93-103.

22. Dietz, WH. Periods of risk in childhood for the development of adult obesity. What do we need to learn? J Nutr 1997; 127: 1884S-1886S.

23. Kuzawa, CW \& Adair, LS. A supply-demand model of fetal energy sufficiency predicts lipid profiles in male but not female filipino adolescents. Eur J Clin Nutr 2004; 58: 438-448.

24. Stettler, N; Bovet, P; Shamlaye, H; Zemel, BS; Stallings, VA; Paccaud, F. Prevalence and risk factors for overweight and obesity in children from Seychelles, a country in rapid transition: the importance of early growth. Int J Obesity 2002; 26: 214-219.

25. Gunnarsdottir, I \& Thorsdottir, I. Relationship between growth and feeding in infancy and body mass index at the age of 6 years. Int $J$ Obesity 2003; 27: 1523-1527.

26. Singhal, A \& Lucas, A. Early origins of cardiovascular disease: is there a unifying hypothesis? Lancet 2004; 363: 1642-1645.

27. Singhal, A; Sadaf-Farooqi, I; O'Rahilly, S; Fewtrell, M; Lucas, A. Early nutrition and leptin concentrations in later life. Am J Clin Nutr 2002; 75: 993-999. 
28. Singhal, A; Fewtrell, M; Cole, TJ; Lucas, A. Low nutrient intake and early growth for later insulin resistance in adolescents born preterm. Lancet 2003b; 361: 1089-1097.

29. Singhal, A; Cole, TJ; Fewtrell, M; Lucas, A. Breastmilk feeding and the lipoprotein profile in adolescents born preterm. Lancet 2004; 363: 15711578.
30. Gillman, MW. Epidemiological challenges in studying the fetal origins of adult chronic disease. Int J Epidemiology 2002; 31: 294-299.

31. Victora, CG \& Barros, FC. Commentary: the catchup dilemma - relevance of Leitch's 'low-high' pig to child growth in developing countries. Int $J$ Epidemiology 2001; 30: 217-220. 